\title{
THE SPECTRUM OF AN $R$-HOMOMORPHISM
}

\author{
A. W. WICKSTEAD \\ (Received 16 September 1975) •
}

\begin{abstract}
Let $E$ be a real Banach space ordered by a closed, normal and generating cone. Suppose also that the order induced on $E$ has the Riesz decomposition property. It is shown that if $T: E \rightarrow E$ is a positive linear operator with the property that $y, z, a \in E$ with $a \geqq T y, T z$ implies there is $x \in E$ with $x \geqq y, z$ and $a \geqq T x$ then the approximate point spectrum and spectrum of $T$ are cyclic subsets of the complex plane. That is, if $\alpha=|\alpha| \gamma$ lies in one of these sets then so does $|\alpha| \gamma^{k}$ for all integers $k$.
\end{abstract}

\section{Introduction}

Throughout this paper $E$ and $F$ will be real Banach spaces ordered by closed, normal and generating cones $E_{+}$and $F_{+}$, for which the induced orders have the Riesz decomposition property (see Alfsen (1971), page 84). This last condition was shown by Ando (1962) to be equivalent to assuming that $E^{*}$ and $F^{*}$, when equipped with the dual orderings, are lattices. The dual space $E^{*}$ (and hence $E^{* *}$ ) can be given an equivalent norm under which it is a Banach lattice. If $x, y \in E$ then $[x, y]$ will denote the order interval $\{z \in R$ : $x \leqq z \leqq y\}$. A linear subspace $I$ of $E$ is an ideal if $x, y \in I$ implies $[x, y] \subset I$ and $I$ is positively generated.

A positive linear operator $T: E \rightarrow F$ is an $R$-homomorphism if for all $y, z \in E$ and $a \in F$ with $a \geqq T y, T z$ there is $x \in E$ with $x \geqq y, z$ and $a \geqq T x$. This notion appears to have originated in Jellett (1968). If $E$ and $F$ are lattices then the $R$-homomorphisms and lattice homomorphisms coincide.

The spectra of positive linear operators on ordered Banach spaces have received considerable attention in recent years (see Schaefer (1974) which we follow for all definitions and notation related to spectra). Abdelaziz (1975) generalised some results known for Banach lattices to the more general spaces considered here. In this note we generalise the much stronger results known for lattice homomorphisms of Banach lattices to $R$-homomorphisms on spaces with the decomposition property. Before doing this we note one more result on positive operators from Schaefer (1974), which can be generalised to this context. We refer the reader to Schaefer (1974), Chapter 
$\mathrm{V}$, definition 4.7 for the definitions of growth condition $(G)$ and a $(G)$-solvable operator (with obvious modifications to apply them to our case).

THEOREM 1. Let E be a Banach space ordered by a closed, normal and generating cone which induces an order with the Riesz decomposition property. Every $(G)$-solvable positive operator on $E$ has cyclic peripheral spectrum.

It is easily verified that a bounded linear operator $T$ satisfies the growth condition $(G)$ if and only if its adjoint, $T^{*}$, does. Let $T$ be a $(G)$-solvable positive operator on $E$, and $\{0\}=E_{0} \subset E_{1} \subset \cdots \subset E_{n}=E$ be a chain of closed $T$-invariant ideals such that each operator $T_{k}$ induced by $T$ on $E_{k} / E_{k-1}$ satisfies $(G)$. The annihilators $E_{k}^{0} \subset E^{*}$ form a chain $\{0\}=$ $E_{n}^{0} \subset E_{n-1}^{0} \subset \cdots \subset E_{0}^{0}=E^{*}$ of closed $T^{*}$-invariant ideals in $E^{*}$. Each quotient $E_{k-1}^{0} / E_{k}^{0}$ may be identified with the dual space $\left(E_{k} / E_{k-1}\right)^{*}$, and the operator induced by $T^{*}$ on $E_{k-1}^{0} / E_{k}^{0}$ with $T_{k}^{*}$, so that it satisfies growth condition $(G)$. Thus $T^{*}$ is $(G)$-solvable. Renorm $E^{*}$ as a Banach lattice (which does not affect spectra) and complexify using Schaefer (1974), Chapter II, definition 11.3 , noting that this does not affect growth condition $(G)$. We may now apply Schaefer (1974), Chapter V, theorem 4.9 to see that the peripheral spectrum of $T^{*}$, and hence of $T$, is cyclic.

\section{2. $R$-homomorphisms}

The key to extending results known for lattice homomorphisms to the case of $R$-homomorphisms is the following result.

THEOREM 2. Let E and F be Banach spaces ordered by closed, normal and generating cones, for which the induced orders have the Riesz decomposition property. If $T: E \rightarrow F$ is an $R$-homomorphism, then $T^{* *}: E^{* *} \rightarrow F^{* *}$ is a lattice homomorphism.

We show that if $\Phi \in F_{+}^{*}$ then $T^{*}[0, \Phi]=\left[0, T^{*} \Phi\right]$. The argument used in the proof of " $(3) \Rightarrow(1)$ " in the theorem in Kim (1975) may then be used to show that $T^{* *}$ is a lattice homomorphism. We must prove that if $G \in$ $\left[0, T^{*} \Phi\right]$ there is $\Psi \in[0, \Phi]$ with $G=T^{*} \Psi$.

Define $\psi$ on $T E$ by

$$
\psi(T x)=G(x) \quad(x \in E) .
$$

$\psi$ is well-defined, for if $T x=T y$ then there are $s, t \in E$ with $s \geqq x-y, 0 \geqq t$ and $0 \geqq T s, T t \geqq 0$ (since $0 \geqq T(x-y), 0$ and $T$ is an $R$-homomorphism). Thus $T s=T t=0$. Now we have

$$
0=\Phi(T s)=\left(T^{*} \Phi\right)(s) \geqq G(s) \geqq 0,
$$


so that $G(s)$, and similarly $G(t)$, are zero. Thus $0=G(s) \geqq G(x-y) \geqq$ $G(t)=0$, and $G(x)=G(y)$. Note also that $\psi$ is positive, for if $T x \geqq 0$ there is $y \geqq x, 0$ with $T x \geqq T y$, so $T x=T y$ and $\psi(T x)=\psi(T y)=G(y) \geqq 0$.

Now define a sublinear functional $p$ on $F$ by the formula

$$
p(a)=\inf \{\Phi(b): b \in F, b \geqq a, 0\} \quad(a \in F)
$$

(this is defined as $\Phi$ is positive and $F$ is positively generated). If $x \in E$ then $\psi(T x) \leqq p(T x)$. This is because if $b \geqq T x, 0$ there is $y \geqq x, 0$ with $b \geqq T y$. Now

$$
\Phi(b) \geqq \Phi(T y) \geqq \psi(T y) \geqq \psi(T x),
$$

and the inequality follows on taking an infimum. By the Hahn-Banach theorem $\psi$ extends to a linear functional $\Psi$ on $F$ with $\Psi(a) \leqq p(a)(a \in F)$. If $a \geqq 0$ then $\Psi(-a) \leqq \inf \{\Phi(b): b \geqq-a, 0\}=0$, so this extension is positive. It is now clear that $\Psi \in[0, \Phi]$ (boundedness being automatic) and that $T^{*} \Psi=$ $G$, so the proof is complete.

It is not known to the author whether or not the converse is true. It is for Markov operators between the spaces of type $A(S)$ considered below.

COROLlary 3. Let $E$ be as in Theorem 1. If $T$ is an $R$-homomorphism on $E$, then the sets $A \sigma(T)$ and $\sigma(T)$ are cyclic.

This follows from applying the corresponding results for lattice homomorphisms in Schaefer (1974), Chapter V, theorem 4.4 and Satz 3.3 of Lotz (1968) to $T^{* *}$, and noting the identities $A \sigma\left(T^{* *}\right)=A \sigma(T)$ and $\sigma\left(T^{* *}\right)=\sigma(T)$.

It is also possible to prove a number of other results in a similar manner. We mention just one. Let $S$ be a Choquet simplex (Alfsen (1971), Chapter 2, $\S 3)$ and $A(S)$ the space of all continuous affine real valued functions on $S$, with the supremum norm and the pointwise partial order. $A(S)^{*}$ is an $A L$-space and $A(S)^{* *}$ an $A M$-space with unit, which may thus be identified with a space $C(X)$. A positive linear operator $T$ on $A(S)$ is a Markov operator if $T 1_{s}=1_{s}$. The Markov $R$-homomorphisms on $A(S)$ (under the name of $R$-homomorphisms) were described in Jellett (1968). If $T$ is a Markov $R$-homomorphism on $A(S)$ then $T^{* *}$ is a Markov lattice homomorphism on $A(S)^{* *}$. Thus the following result follows from Wolff (1969), theorem 2.2 .

Corollary 4. If $S$ is a Choquet simplex and $T$ a Markov $R$ homomorphism on $A(S)$, then $\sigma(T)$ is either a cyclic subset of $\{\lambda \in C$ : $|\lambda|=1\} \cup\{0\}$ or the whole of $\{\lambda \in C:|\lambda| \leqq 1\}$. 


\section{References}

N. H. Abdelaziz (1975), 'Spectral properties of some positive operators in a Banach space with the decomposition property'. Proc. Amer. Math. Soc. 48, 344-350.

E. M. Alfsen (1971), Compact Convex Sets and Boundary Integrals (Springer-Verlag, 1971).

T. Ando (1962), 'On the fundamental properties of a Banach space with a cone'. Pacific J. Math. $12,1163-1169$.

F. Jellett (1968), 'Homomorphisms and inverse limits of Choquet simplexes'. Math. Z. 103, 219-226.

J. Kim (1975), 'The characterisation of a lattice homomorphism'. Can. J. Math. 27, 172-175.

H. P. Lotz (1968), 'Über das Spektrum positiver Operatoren'. Math. Z. 108, 15-32.

H. H. Schaefer (1974), Banach Lattices and Positive Operators (Springer-Verlag, 1974).

M. Wolff (1969), 'Über das Spektrum von Verbandshomomorphismen in $C(X)$ '. Math. Ann. 182, 161-169.

Department of Pure Mathematics, The Queen's University of Belfast, Northern Ireland. 